\title{
Piotr Gurgul*
}

\section{New Mobile Marketing Capabilities of the Android Platform}

\section{Introduction}

The emergence of smartphones, tablets and other intelligent mobile devices proved to bring revolutionary ideas and tools for smart marketing. Moreover, the advances in telecommunications leading to a phenomenon called service convergence [21], will potentially make the mobile internet the sole and uniform communication medium. In fact, by the end of 2014, there will be more mobile internet users than 'desktop internet' users [18]. This change is shown in Figure 1, which extrapolates the existing trends to 2015. Such behavior adheres to the rules of network economy summarized for the first time by Kelly in 1997 [14]. Disruptive innovations are changing the mobile market faster than ever and there is a wide choice of brand new solutions that can revolutionize completely the way mobile devices are used currently for marketing purposes. The need for such new solutions has been voiced in [2], [4] and [13].

Moreover, the smartphone market size and value have risen tremendously over the past few years, making it very attractive for advertisers. It is no longer an experimental niche [17]. Figure 2 shows the percentage of mobile media users among citizens of eight selected, developed countries.

However, most businesses allocate only a small percentage of their marketing budgets to this form of promotion. Even if they already apply mobile marketing, its use is mostly limited to very basic features, such as plain SMS advertising.

Nevertheless, smartly leveraged mobile media can help to increase the quality of advertising and therefore boost conversion rates together with improving metrics such as cost per acquisition, cost per click or cost per loyal user.

* AGH University of Science and Technology, Department of Computer Science, email:pgurgul@agh.edu.pl 


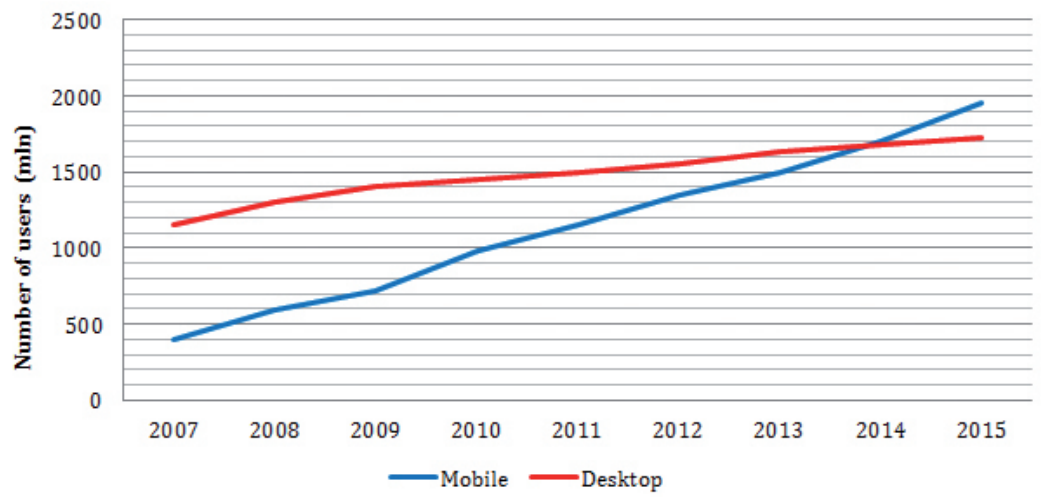

Figure 1. Anticipated increase of mobile and desktop internet users Source: own elaboration based on [18]

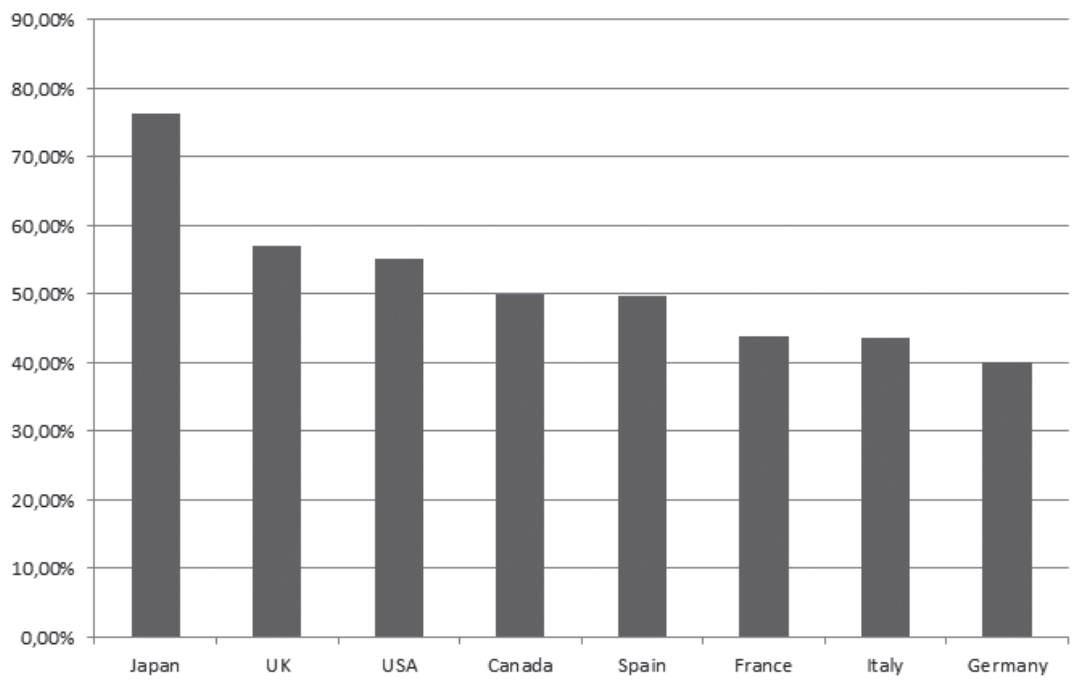

Figure 2. Penetration of markets by mobile media in selected countries Source: own elaboration based on [5]

Smartphones definitely favor more intelligent and widely understood mobile marketing. Usually equipped with numerous gadgets and features like barcode scanners, front camera or GPS navigation they are even better than computers 
at discovering and satisfying their owners' needs. Moreover, they tend to be far more personal devices than ordinary mobile phones used to be. This is easily explicable, since nowadays they carry much more sensitive information than just text messages or call logs. A smartphone is aware of a user's e-mails, location, social network activities etc. As a result, aggressive marketing methods such as spamming will be perceived even less tolerantly than it is in the case of e-mails. Using mobile media involves accepting both up and downsides.

Konkol [15] emphasized two most important properties of mobile media:

- Mobile media are rich and allow communication in many different configurations.

- They provide return channel that is integrated with other communication tools.

A convenient return channel facilitates not only user response to call to action statements, but is perfectly suitable for more personalized and complicated dialogues. In the age of traditional mobile phones, the only implementation of this channel were text messages. Smartphones have introduced more a flexible use of them by letting in external applications with internet access.

This paper aims to cover the existing and possible future impact of the new technical features of smartphones and other intelligent mobile devices. Special emphasis is put on the Android Operating System since its business model is fully bound to revenues from mobile advertising.

The structure of this paper is as follows: section 2 presents an overview of Android seen as a marketing platform. Section 3 introduces Android 4.0 Ice Cream Sandwich and the changes introduced. Section 4 focuses on a new way of shortrange communication that is very attractive for smartphones (NFC). Section 5 describes metrics for measuring effectiveness and efficiency of mobile marketing. The last section concludes this paper.

\section{Android as a mobile marketing platform}

Like most IT companies, Android Inc. was founded in Sillicon Valley (Palo Alto) by Andy Rubin, Rich Miner, Nick Sears and Chris White in 2003. Rubin described his mission as creating “(...) smarter mobile devices that are more aware of its owner's location and preferences” [3]. The company operated secretly until it was bought by Google in 2005. Then it started to be clear that Google aimed to enter the mobile market. Unlike its competitors (like BlackBerry or iPhone see [12]), Google decided to make the platform free and open to enable wide adoption. In the light of Google's sources of income, this is fully understandable. 
Although Google sells numerous additional services such a corporate Gmail and Google Docs, Google Earth professional edition or Google Voice credits, their share in company total revenues is less than one percent. The remaining part comes from advertising.

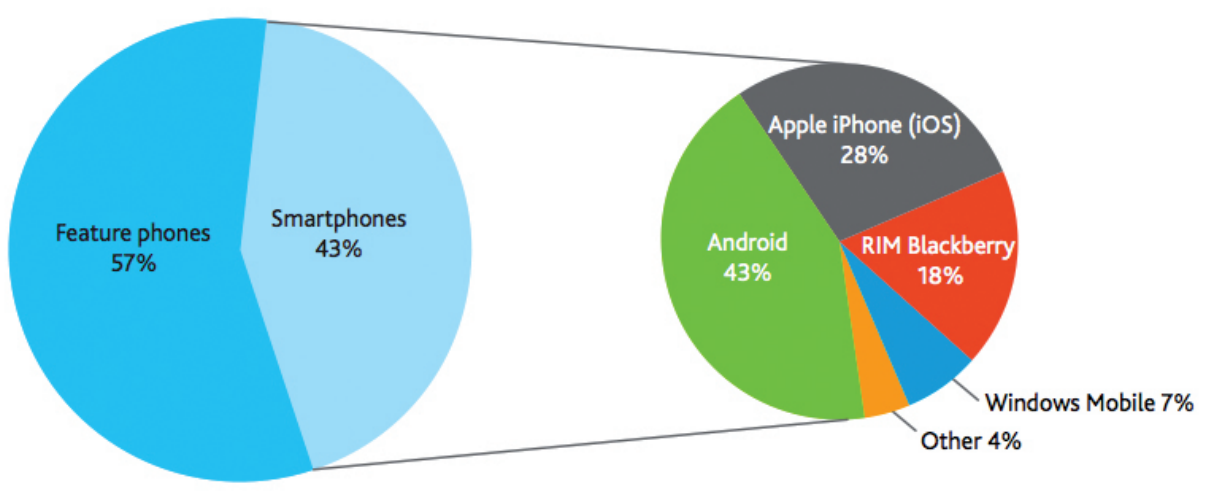

Figure 3. Mobile OS market share

Source: [8]

Using an Android device requires the possession of a Google account, which in most cases implies choosing GMail as e-mail service provider. This allows Google not only to extend their advertising infrastructure by mobile devices, but also to acquire new users on the stationary internet. Having users signed in while browsing the internet allows more accurate targeting of advertising content. Another way of increasing mobile advertising space is cooperation with external publishers. This is achieved by allowing third-party entities to release either paid or free applications in Google's Play Store. Many of them earn on mobile ads that are provided by Google (partner network). The count of applications available in Play Store has already exceeded 550,000 [9]. Since its commercial beginning in 2008 , Android has generated $\$ 550 \mathrm{M}$ in revenues alone [1], which pales into insignificance compared to $\$ 38 \mathrm{~B}$ Google revenues only for 2011 . However, mobile advertising earnings are rising very sharply, so this proportion may change dramatically in the future. In terms of market share, Android has already won pole position. Figure 3 shows the Android market share as of Q4 2011. Figure 4 confirms that Android is also a leader in terms of share of the total number of impressions of the smartphone-displayed advertising. 


\section{New technical features}

Android has lot to offer to mobile marketers. The most commonly used features are the simplest ones - mobile banners, context ads and sponsored applications. There have been also several attempts to utilize more advanced capabilities like Bluetooth advertising, geospatial ads, barcode scanners, augmented reality, sponsored widgets and QR codes.

\section{- Android $\|$ iOS $\|$ Blackberry $\|$ Other $\|$ Windows Phone $\|$ Symbian}

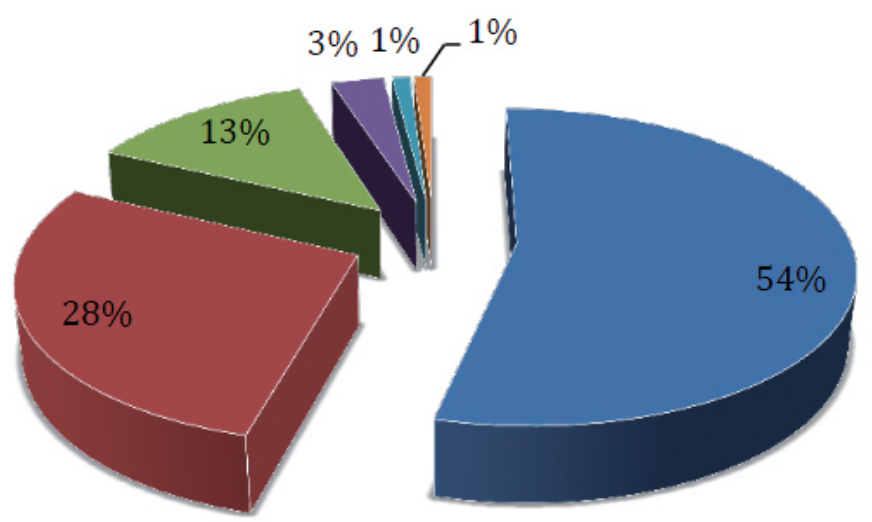

Figure 4. Share of various mobile OS in total number of mobile ads impressions Source: own elaboration based on [11]

Android 4.0 Ice Cream Sandwich (ICS) was released on October 19, 2011. It is already in its eighth publicly available version. It contains a wide range of improvements and redefinitions of already present features.

One of the most visible changes comes in terms of Graphical User Interface (GUI). It offers increased usability and elegance. There are new, virtual buttons located in the bottom action bar that serve for most common actions like starting a phone call, opening the address book, sending a text message or opening the web browser. As a matter of fact, the entire graphic system has been redefined to fit the needs of high-resolution screens (e.g. AMOLED). The author has tested and compared three devices running different versions of Android (HTC myTouch - Android 1.6, Google Nexus One - Android 2.2, Samsung Galaxy Nexus - 4.0) and the supremacy of the latter one is clearly visible. 
Another feature that increases usability are resizable widgets. Now, it is easy not only to make shortcuts on Android desktops, but also to embed fragments of entire applications on the desktop. This is an impressive quality change that allows us to e.g. check for new e-mails and social streams without entering any app.

There has been also introduced a 'favorites tray' that allows dragging and dropping applications and shortcuts for quicker access. While unlocking the screen it is also easy to enter the camera view.

Google also put great effort into improving the e-mail application experience. The improved auto-completion of recipients is definitely worth mentioning. And, in line with what has been mentioned in previous paragraphs, e-mail app can be dragged as a widget.

Receiving and handling incoming calls in ICS is also significantly easier. When the phone rings, now it is possible to respond with a text message even without unlocking the screen. There are also some predefined message templates that mean spending minimum time when busy.

The camera software and photo gallery have been also greatly improved, adding some extra features like panorama mode and built-in face detection. Moreover, quick photo sharing capabilities have been mastered.

As promised by its authors, Android ICS introduces better web browsing experience. The speedup of webpage rendering is now $550 \%$ better than its previous version [22]. It is also possible to request a 'desktop' version of a web-page and browse it using easy zoom-in and zoom-out commands.

One of the key security innovations is also a face recognition system for unlocking the screen. The front camera compares an actual face with a saved pattern and decides, if a given face is similar enough to grant access. If this is not the case, the user still can type his PIN to unlock the phone. Unfortunately, this is still more like an experimental feature, since its performance highly depends on current light conditions and angle. According to this author's experience, it usually takes between 2-3 attempts to unlock the phone in bright surroundings and it is virtually impossible when it is dark. This still makes the visual pattern more comfortable alternative.

One of the biggest innovations introduced in ICS is Android Beam for NFC-based sharing. NFC stands for Near-Field Communication and constitutes a set of standards for smartphones that enable easy communication between nearby devices. This communication includes (but is not limited to) contactless transactions and data exchange. The maximum range is just a few centimeters. Apparently, NFC serves not only for communication between devices, but can be used also to exchange data with an unpowered chip, called a 'tag'. Countless existing and prospective marketing commercial applications of NFC will be explored in 
the next section. Android Beam is an application allowing rapid exchange of data for two NFC-enabled Android devices placed nearby. Such an exchange can include favorite applications, movies, files, contacts, pictures or even comparing game scores.

Android supports Wi-Fi Direct which allows peer-to-peer connection between two nearby (not necessarily Android) devices and instant sharing of various content. Such a connection is completely adhoc and does not require any structured Wi-Fi network (e.g. a router).

Lastly, Android ICS supports one more, but rather specialized way of communication. It is called the Bluetooth Health Device Profile (Bluetooth HDP) and provides easy access to wireless medical devices or sensors present in hospitals and fitness centers. As an example, an Android 4.0 phone can obtain and interpret results from a HDP-ready blood pressure monitor.

\section{Commercial use of Near Field Communication}

Although NFC Forum was established by Nokia, Phillips and Sony as early as 2004 , the Near Field Communication standard is relatively young and poorly explored, especially in marketing. For now, its primary commercial application has been developed by MasterCard to introduce a software PayPass function for smartphones that requires neither a PayPass card nor a sticker.

Although mobile payments are currently the most common way of using NFC worldwide, marketing applications will certainly follow, because in terms of marketing functionality, NFC is no different from QuickResponse (QR codes) but easier to use. QR codes require enabling camera mode or barcode scanner and code scanning, while an NFC exchange does not require any special action. So far, many businesses use these two technologies alongside each other.

The number of places and items displaying QR codes is skyrocketing and now it is safe to say they are ubiquitous. QR codes, that were once only industry standards for monitoring vehicles during assembly, now serve primarily for marketing. This was caused by dissemination of smartphones and other intelligent devices equipped with a camera and barcode scanner. The main advantage of using a QR code is the fact that they provide virtually effortless access to promotional content like a company website or advertisement. This obviously boosts conversion rates.

NFC stands somewhere between QR codes and Bluetooth advertising. The latter turned out to be too expensive and to raise many privacy concerns. Bluetooth proximity marketing, called sometimes 'Bluecasting' was used in just a few dozen major campaigns. 
The marketing use of NFC, QR codes and Bluetooth can be divided by subject into several categories:

- Retailers may use this way of communication for upselling, when the customer is already in the store.

- Transponders in malls or markets may offer free maps and guides, together with special offers and ads 'smuggled' into the content.

- Sports Arenas where free wallpapers or other gadgets can come along with marketing communications.

One of the earliest NFC-ready applications available in Play Store is a project called EnableTable. For now, it was designed exclusively for Android and serves for the smart increasing of restaurant's customer loyalty. The basic idea behind this application is simple. When a server brings the bill to the guest table, it is equipped with a special tag that emits NFC data. The data contains a discount 'thank you' coupon which is downloaded to the user's phone upon payment. The most common pattern that the coupon offers is $10-15 \%$ off the next payment, which should encourage customers to return. However, the remaining options may be much more sophisticated ranging from a free-drink coupon through free tunes to contests. Understandably, these kinds of offers are nothing new in the marketing world but their existing implementations are somehow inconvenient for customers. It is easy to lose a paper coupon, forgot it or let it expire. Having an e-coupon as a mobile application makes it self-manageable and smart. EnableTable notifies a user when one of the coupons is about to expire. Currently, the main obstacle against the wide adoption of this solution is the small number of participating restaurants. Figure 5 shows the EnableTable functionality scheme.
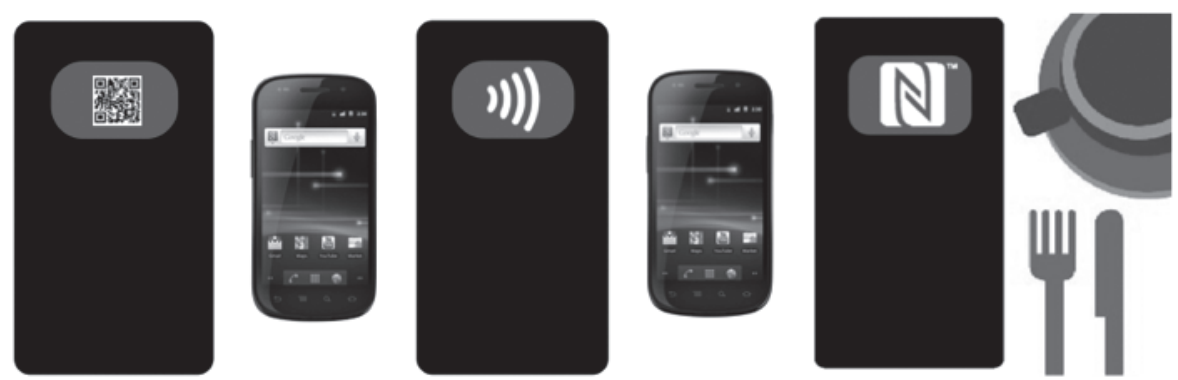

The "N" logo is a trademark of the NFC Forum, Inc in the United States and in other countries. The Nexus S smartphone is co-developed by Google and Samsung

Figure 5. EnableTable functionality scheme

Source: [6] 


\section{Measuring results}

The aim of this paragraph is to compare and contrast the effectiveness and efficiency of mobile marketing as opposed to internet marketing.

The most common metrics for effectiveness are [16]:

- Reach $\mathbf{r}$. The number of unique visitors that have seen the ad.

- Frequency $f$. The average number of times that a recipient has seen the given ad.

- Click Through Rate (CTR). The percentage of visitors that have seen the ad and followed its content (e.g. clicked).

- Conversion Rate. The percentage of visitors that see the ad and take the desired action (e.g. register or make a purchase).

The MediaMind organization [7] has investigated 230M impressions of internet ads both in traditional and mobile web browsers. It turned out that CTR for mobile ads was $0.61 \%$ as opposed to only $0.07 \%$ for traditional web ads. These are not only much better results, but most likely, also underestimated. This is due to the 'offline effect' [23], which says that some mobile users just perform the desired action instead of following the ad. As an example, a user walking down the street and reading news on his smartphone spots a sponsored sandwich bar nearby (geospatial advertising, see [20], [23]). Instead of following the ad, the user just goes and visits the sandwich bar. Understandably, this is very rarely the case with desktop computers.

However, critics indicate that some of these clicks may be caused just by accidental tapping of the touchscreen. Mobile phone displays are much smaller and therefore mobile ads occupy relatively more space.

Efficiency parameters can be described in detail by:

- Cost per Click - determines the price paid by the advertiser when a user follows (clicks) the ad.

- Cost per Mille - determines the price paid by the advertiser for one thousand impressions of the ad.

- Cost per View - determines the price paid by the advertiser when the advertised content is displayed to the user. Used for the Pay per View model, mostly for video ads.

- Cost per Acquisition - determines the price paid by the advertiser when a user performs the desired action. Used as a basic payment model for affiliate marketing.

As of March 2012, the average Cost per Click for smartphones was $\$ 0.53$ as opposed to $\$ 0.83$ for desktop computers [10]. 


\section{Best practices and conclusions}

Although mobile marketing is still a very young and poorly explored discipline, there are already many guides and manuals available that indicate what makes mobile marketing both successful and efficient.

First of all, mobile phones are treated as very personal, and sometimes even intimate devices, which users are unwilling to share or lend. For this reason, it is much more difficult to persuade a user to accept promotional content. In the first place, it need to be either useful or entertaining. Broadcasting mass-media style advertising through mobile media often ends up in customer ignorance and very low acceptance rates.

It is crucial to provide an honest, clear and attractive Call to Action [15]. This can be either in the form of discount code, free coffee or useful sponsored application.

It is also very important to be aware of the diversity of different devices when planning a campaign [19]. Moreover, communication over mobile media cannot assume definite connection parameters, since they vary.

Mobile marketing on smartphones is definitely a powerful and promising, yet strongly underrepresented means of smart promotion. The numerous technical features useful for marketing seem at least partly undiscovered. There are many attempts to try new possibilities, but only few of them find followers (as happened with e.g. QR codes).

The aim of this paper was to show the raising significance of the Android platform and numerous opportunities brought to marketers by its emergence. Focusing mostly on the newest solutions available, NFC has been explored in detail. Judging by the early results, this is one of the ways to go.

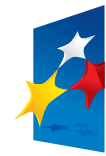

KAPITAt LUDZKI

NARODOWA STRATEGIA SPÓJNOŚC

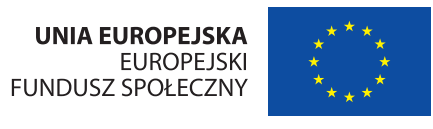

The work described in this paper was partially supported by The European Union by means of European Social Fund, PO KL Priority IV: Higher Education and Research, Activity 4.1: Improvement and Development of Didactic Potential of the University and Increasing Number of Students of the Faculties Crucial for the National Economy Based on Knowledge, Subactivity 4.1.1: Improvement of the Didactic Potential of the AGH University of Science and Technology "Human Assets", No. UDA - POKL.04.01.01-00-367/08-00. 


\section{References}

[1] Arthur C., Google's Android has generated just \$550m since 2008, figures suggest, "The Guardian", 30.03.2012.

[2] Duncan T., Everett S., Client perceptions of integrated communications, "Journal of Advertising Research", 1993, vol. 3(32), pp. 30-39.

[3] Elgin B., Google Buys Android for Its Mobile Arsenal, "Bloomberg Businessweek", 2005, nr 25.

[4] Hughes M., Buzzmarketing: Get People to Talk About Your Stuff, "Portfolio Hardcover", 2005.

[5] http://www.comscore.com.

[6] http://www.enabletable.com.

[7] http://www.mediamind.com.

[8] http://www.nielsen.com.

[9] http://play.google.com.

[10] http:/www.simplyzesty.com/mobile/smartphones-will-generate-25-of-paidsearch-ads-click-by-dec-2012/.

[11] http://www.techcrunch.com.

[12] Isaacson W., Steve Jobs, "Simon \& Schuster", 2011.

[13] Kaplan A., If you love something, let it go mobile: Mobile marketing and mobile social media 4 x 4, "Business Horizons", 2012, 55(2), pp. 129-139.

[14] Kelly K., New rules for the new economy, "Wired", 1997.

[15] Konkol S., Marketing mobilny. "Helion", 2009.

[16] Kotler P., Armstrong G., Wong V., Saunders John A., Principles of Marketing, "Pearson", 2006.

[17] Leppaniemi M., Mobile marketing communications in consumer markets, "Acta Oeconomica", University of Oulu, 2008.

[18] Mobile Internet Report, Morgan Stanley, 2011, http://www.morganstanley. com/institutional/techresearch.

[19] Schultz D., The next generation of integrated marketing communication, "Interactive Marketing", 2003, vol. 4(4), pp. 318-319.

[20] Sharma C., Herzog J., Melfi V., Mobile Advertising - Supercharge, Your Brand In the Exploding Wireless Market, "John Wiley \& Sons", 2008.

[21] Van Dijk J., The network society, "Sage Publications", 2008.

[22] V8 benchmark, http://www.android.com.

[23] Ziemiński A., Gamdzyk M., Telefon wszechstronnie marketingowy, „Marketing w Praktyce", 2009 (5), pp. 43-46. 